\title{
Diffusion-Controlled Electron Transfer Processes and Power-Law Statistics of Fluorescence Intermittency of Nanoparticles
}

\author{
Jau Tang* and R. A. Marcus ${ }^{\dagger}$ \\ Noyes Laboratory of Chemical Physics, California Institute of Technology, Pasadena, California 91125, USA
}

(Received 7 April 2005; published 29 August 2005)

\begin{abstract}
A mechanism involving diffusion-controlled electron transfer processes in Debye and non-Debye dielectric media is proposed to elucidate the power-law distribution for the lifetime of a blinking quantum dot. This model leads to two complementary regimes of power law with a sum of the exponents equal to 2 , and to a specific value for the exponent in terms of a distribution of the diffusion correlation times. It also links the exponential bending tail with energetic and kinetic parameters.
\end{abstract}

DOI: 10.1103/PhysRevLett.95.107401

PACS numbers: 78.67.Bf, 73.21.La, 73.63.Kv, 78.67.Hc

Recent advances in nanoscience and nanotechnology and their potential applications have generated wide interest. The development of techniques in probing single molecules has provided a tool to study its intrinsic properties and its interaction with the surroundings. One of the unusual phenomena observed in nanoparticles is fluorescence intermittency of quantum dots and the power-law statistics for the duration time for the "on" an "off" events [1-10]. Supplementing previous theoretical studies [6,7,11-16], a mechanism is provided in this Letter to elucidate these phenomena. This model involves diffusioncontrolled charge transfer processes in energy or configuration space $[17,18]$. For fast diffusion, the model yields the well-known simple exponential decay. However, in the regime of slow diffusion, the model leads naturally to a power-law behavior. To be more general, we consider anomalous diffusion in a non-Debye dielectric medium with a distribution of diffusion correlation times. There exists in the literature two approaches, partial ordering prescription (POP) with a time-dependent but nonretarded diffusion coefficient, and chronological ordering prescription (COP) with convolution of a time-retarded diffusion kernel [19]. POP is more commonly used in treating electron transfer reactions [20], These diffusion-controlled reaction models provide physical insight into the specific value of the exponent, a reason for the bending tail at longer times, and the connection of the bending factor to the activation energy of the electron transfer rate constant.

In this work, one models stochastic processes in the energy or configuration space that represent the fluctuating interactions of a probe (a single molecule or a quantum dot) with its surrounding heat bath (supporting substrate or anchored organic molecules). We consider a POP type 1D non-Markovian equation, with a population sink at the potential energy crossing $\left(Q=Q_{c}\right)$ between $U_{1}(Q)$ for the "light" state $|1\rangle$ and $U_{2}(Q)$ for the "dark" state $|2\rangle$. One has

$$
\begin{aligned}
\frac{\partial}{\partial t} \rho_{k}(Q, t)= & D_{k}(t) \frac{\partial}{\partial Q}\left(\frac{\partial}{\partial Q}+\frac{1}{k_{B} T} \frac{\partial}{\partial Q} U_{k}(Q)\right) \rho_{k}(Q, t) \\
& -\frac{2 \pi\left|V_{k}\right|^{2}}{\hbar} \delta\left(U_{1}(Q)-U_{2}(Q)\right) \rho_{k}(Q, t) .
\end{aligned}
$$

One can obtain the Green function for sink-free diffusion in a harmonic potential $\kappa q^{2} / 2$ as [20]

$$
\begin{aligned}
G_{k}\left(q, q^{\prime} ; t\right)= & \frac{1}{\sqrt{2 \pi \Delta_{k}^{2}\left(1-\Theta_{k}^{2}(t)\right)}} \\
& \times \exp \left[-\frac{\left(q-q^{\prime} \Theta(t)\right)^{2}}{2 \Delta_{k}^{2}\left(1-\Theta_{k}^{2}(t)\right)}\right],
\end{aligned}
$$

where the diffusion constant $D_{k}(t)$ is related to the dielectric response function $\bar{\chi}(s)$ and the dielectric permittivity $\bar{\varepsilon}(s)$ by [20]

$$
\begin{aligned}
D_{k}(t)=-\Delta_{k}^{2} \frac{d}{d t} \ln \left(\Theta_{k}(t)\right) & \bar{\Theta}_{k}(s)=\frac{1}{s+\left[1 / \bar{\tau}_{L, k}(s)\right]}, \\
\bar{\tau}_{L, k}(s)=\frac{\varepsilon_{\infty}}{\varepsilon_{0}} \frac{1-\bar{\chi}_{k}(s)}{s \bar{\chi}_{k}(s)}, & \bar{\chi}_{k}(s)=\frac{\bar{\varepsilon}_{k}(s)-\varepsilon_{\infty}}{\varepsilon_{0}-\varepsilon_{\infty}} .
\end{aligned}
$$

For a Debye medium $\bar{\chi}(s)=1 /\left(1+s \tau_{D}\right)$, one has $\bar{\tau}_{L, k}(s)=\tau_{L, k} \equiv \tau_{D, k} \varepsilon_{\infty} / \varepsilon_{0}, \Theta_{k}(t)=\exp \left(-t / \tau_{L, k}\right)$, and a time-independent diffusion constant $D(t)=\Delta_{k}^{2} / \tau_{L, k}$ as considered in our previous study [16], whereas for a Cole-Davison (CD) dielectric medium [21] $\bar{\chi}(s)=1 /(1+$ $\left.s \tau_{D}\right)^{\beta_{\mathrm{CD}}}$, diffusion constant is time dependent.

It can be shown from Eqs. (1b) and (1c) that in the short time regime $\left(t \ll \tau_{L}\right.$, and $\left.\tau_{D}\right)$ the mean square displacement $\quad \sigma^{2}(t)=\left\langle(Q(t)-\langle Q(t)\rangle)^{2}\right\rangle \approx 2\left(t / \tau_{D}\right)^{\beta_{\mathrm{CD}}} \Delta^{2} \tau_{D} /$ $\tau_{L} \Gamma\left(1+\beta_{\mathrm{CD}}\right)$. At times $t$ much longer than the diffusion correlation time, however, $\sigma^{2}(t)$ becomes a constant as the system approaches thermal equilibrium. The asymptotic behavior of $\sigma^{2}(t)$ at both short and long time limits have also been obtained previously by Metzler et al. [22], using a different approach with a fractional Fokker-Planck equation. 
Because each blinking event starts at the energy-level crossing, one has the initial condition $\rho_{\kappa}(Q, 0)=\delta(Q-$ $\left.Q_{c}\right)$. The blinking statistics $P_{\text {on }}(t)$ for the on events of a neutral quantum dot (QD) [or $P_{\text {off }}(t)$ for the off events of a dark QD] is defined as the waiting time distribution function for a QD that is initially in the neutral light state (or dark state) and is turned into the charged "dark state" (or light state) between $t$ and $t+d t$ per unit $d t$. Using the Green function method, the Laplace transform of $P(t)$ can be derived from Eq. (1) as

$$
\begin{aligned}
\bar{P}_{k}(s) & =-\int_{0}^{\infty} d t e^{-s t} \frac{d}{d t}\left(\int_{0}^{\infty} d Q \rho_{k}(Q, t)\right) \\
& =\frac{A_{k} \bar{G}_{k}\left(Q_{c}, Q_{c} ; s\right)}{1+A_{k} \bar{G}_{k}\left(Q_{c}, Q_{c} ; s\right)} \\
A_{k} & =\frac{2 \pi}{\hbar}\left|V_{k}\right|^{2}\left|\partial\left(U_{1}(Q)-U_{2}(Q)\right) / \partial Q\right|_{Q=Q_{c}} .
\end{aligned}
$$

For simplicity, diffusion on harmonic potentials $U_{1}(Q)=\kappa\left(Q-Q_{0,1}\right)^{2} / 2 \quad$ and $\quad U_{2}(Q)=\kappa(Q-$ $\left.Q_{0,2}\right)^{2} / 2+\Delta G^{0}$ is considered, where $\kappa$ is the force constant $\kappa \Delta_{k}^{2}=k_{B} T, \Delta G^{0}$ the free energy gap, and the reorganization energy $\lambda=\kappa\left(Q_{0,1}-Q_{0,2}\right)^{2} / 2$. In the short time limit, Eq. (1b) yields

$$
\begin{aligned}
A_{k} G_{k}\left(Q_{c}, Q_{c} ; t\right)= & \frac{2 \pi\left|V_{k}\right|^{2} / \hbar}{\sqrt{4 \pi \lambda k_{B} T\left(1-\Theta_{k}^{2}(t)\right)}} \\
& \times \exp \left[-\frac{\left(Q_{c}-Q_{0, k}\right)^{2}\left(1-\Theta_{k}(t)\right)}{2 \Delta_{k}^{2}\left(1+\Theta_{k}(t)\right)}\right] \\
\approx & \frac{1}{\Gamma\left(1-\beta_{\mathrm{CD}} / 2\right) t_{c, k}}\left(t / t_{c, k}\right)^{-\beta_{\mathrm{CD}} / 2} \\
& \times \exp \left(-(\boldsymbol{\Gamma} t)^{\left.\beta_{\mathrm{CD}}\right)} \text { if } t \ll \tau_{D}, \tau_{L}, k\right.
\end{aligned}
$$

or

$$
\begin{aligned}
A_{k} \bar{G}_{k}\left(Q_{c}, Q_{c} ; s\right) \approx & \left(\left(s+\Gamma_{k}\right) t_{c, k}\right)^{1-\beta_{\mathrm{CD}} / 2}, \\
& \text { if } s \geq \Gamma_{k}, \quad \beta_{\mathrm{CD}} \sim 1
\end{aligned}
$$

where we defined two parameters $t_{c, k}$ and $\Gamma_{k}$ as

$$
\begin{aligned}
\left(t_{c, k} / \tau_{D, k}\right)^{\beta_{\mathrm{CD}}} / t_{c, k}^{2} & \equiv \pi\left|V_{k}\right|^{4} \tau_{L, k} \Gamma\left(\beta_{\mathrm{CD}}+1\right) / 2 \lambda k_{B} T \hbar^{2} \tau_{D, k} \\
\left(\Gamma_{k} \tau_{D, k}\right)^{\beta_{\mathrm{CD}}} & \equiv\left(Q_{c}-Q_{0, k}\right)^{2} \tau_{D, k} / 4 \Delta_{k}^{2} \tau_{L, k} \Gamma\left(\beta_{\mathrm{CD}}+1\right),
\end{aligned}
$$

and $\Gamma\left(\beta_{\mathrm{CD}}+1\right)$ is the gamma function. From Eqs. (2) and (3) one obtains

$$
\bar{P}_{k}(S) \approx \frac{1}{1+\left(\left(s+\Gamma_{k}\right) t_{c, k}\right)^{1-\beta_{\mathrm{CD}} / 2}} .
$$

For the normal diffusion case in a Debye medium with $\beta_{\mathrm{CD}}=1$, from the inverse Laplace transform of Eq. (4), one obtains

$$
\begin{aligned}
P_{k}(t)= & \frac{1}{\sqrt{\pi t_{c, k} t}}\left[1-\sqrt{\pi t / t_{c, k}} \exp \left(t / t_{c, k}\right) \operatorname{erfc}\left(\sqrt{t / t_{c, k}}\right)\right] \\
& \times \exp \left(-\Gamma_{k} t\right)
\end{aligned}
$$

as illustrated in Fig. 1 and

$$
\begin{aligned}
P_{k}(t) & \sim \frac{1}{\sqrt{\pi t_{c, k}}} t^{-1 / 2} \quad \text { if } t \ll t_{c, k}, \\
P_{k}(t) & \sim \frac{\sqrt{t_{c, k}}}{2 \sqrt{\pi}} t^{-3 / 2} \exp \left(-\Gamma_{k} t\right) \quad \text { if } t_{c, k} \ll t \ll \tau_{L, k} .
\end{aligned}
$$

Equations (5) and (6) were obtained previously [16]. From Eq. (3b), one can relate $\Gamma_{k}$ to $E_{a, k}$, the energy difference between the potentials at the crossing and the bottom, by $\Gamma_{k}=E_{a, k} / 2 \tau_{L, k} k_{B} T$, where $E_{a, \text { on }}=\kappa\left(Q_{c}-Q_{0,1}\right)^{2} / 2=$ $\left(\lambda+\Delta G^{0}\right)^{2} / 4 \lambda$ and $E_{a, \text { off }}=\kappa\left(Q_{c}-Q_{0,2}\right)^{2} / 2=$ $\left(\lambda-\Delta G^{0}\right)^{2} / 4 \lambda$. The bending tail observed for the on events [4] implies a large $E_{a, \text { on }}$ or a short $\tau_{L, I}$ for the forward reaction. Because diffusion is light driven [23], $\tau_{L, I}$ decreases at higher light intensity and the bending factor increases. At small $s\left(t \gg \tau_{k}\right), A_{k} \bar{G}_{k}\left(Q_{c}, Q_{c} ; s\right) \approx$ $\gamma_{k} / s+f_{k}$ and Eq. (2) yields

$$
\begin{gathered}
P_{k}(t) \sim \frac{\gamma_{k, \text { eff }}}{1+f_{k}} \exp \left(-\gamma_{k, \text { eff }} t\right), \quad \gamma_{k, \text { eff }}=\frac{\gamma_{k}}{1+f_{k}}, \\
\gamma_{k} \equiv\left(A_{k} / \sqrt{2 \pi \Delta_{k}^{2}}\right) \exp \left(-E_{a, k} / k_{B} T\right),
\end{gathered}
$$

where $\gamma_{1}$ and $\gamma_{2}$ are the nonadiabatic forward and backward rate constants and $f_{k} \equiv \int_{0}^{\infty} d t\left[A_{k} \exp \left(-2 \Gamma_{k} \tau_{L, k} \times\right.\right.$ $\left.\left.\tanh \left(t / 2 \tau_{L, k}\right)\right) / \sqrt{2 \pi \Delta_{k}^{2}\left(1-\exp \left(-2 t / \tau_{L, k}\right)\right)}-\gamma_{k}\right]$. Equation (6) indicates a power-law distribution with an exponent at $-1 / 2$ at very short time which changes later to $-3 / 2$ with an exponential damping tail, and finally becomes the single exponential in Eq. (7) at a much longer time.

For the anomalous diffusion case, as in a Cole-Davidson dielectric medium [21] with $\beta_{\mathrm{CD}} \neq 1$, the inverse Laplace transform of Eq. (4) is related to the time derivative of the Mittag-Leffler function [24]

$$
P_{k}(t) \approx \frac{d}{d t}\left[E_{-1+\beta_{\mathrm{CD}} / 2}\left(-\left(t / t_{c, k}\right)^{-1+\beta_{\mathrm{CD}} / 2}\right)\right] \text { if } t \ll 1 / \Gamma_{k}
$$

where $E_{a}(z)=\sum_{n=0}^{\infty} z^{n} / \Gamma(n a+1)$. Two complementary power-law regimes are illustrated in Fig. 2 and

$$
\begin{aligned}
P_{k}(t) & \sim \frac{1}{\Gamma\left(1-\left(\beta_{\mathrm{CD}} / 2\right)\right) t_{c, k}}\left(t / t_{c, k}\right)^{-\beta_{\mathrm{CD}} / 2} \quad \text { if } t \ll t_{c, k} \\
P_{k}(t) & \sim \frac{1}{\left|\Gamma\left(\left(\beta_{\mathrm{CD}} / 2\right)-1\right)\right| t_{c, k}}\left(t / t_{c, k}\right)^{-2+\beta_{\mathrm{CD}} / 2} \\
& \text { if } t_{c, k} \ll t \ll 1 / \Gamma_{k,} \tau_{L, k} .
\end{aligned}
$$

The above results correspond to the first passage approxi- 


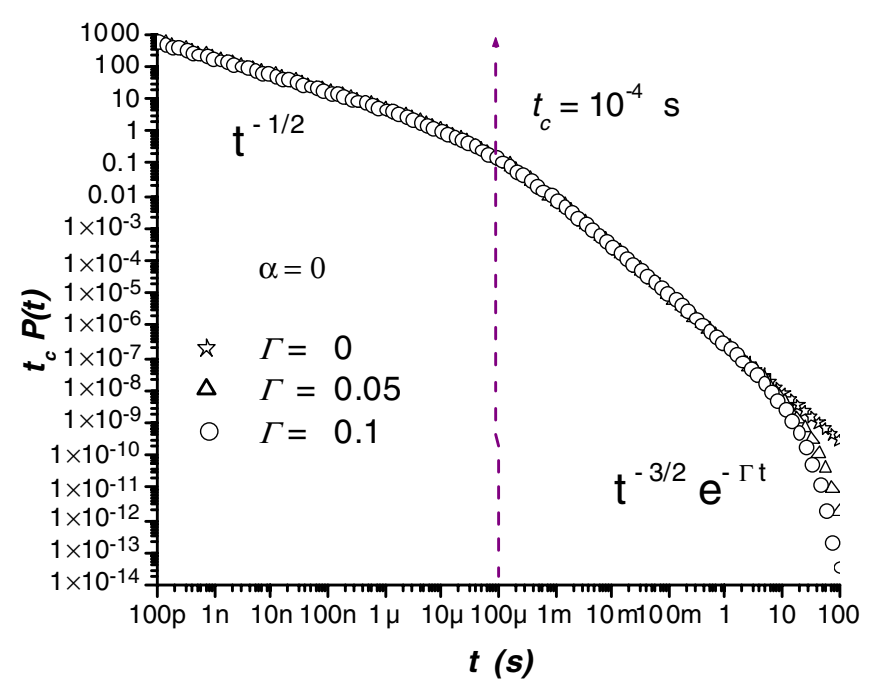

FIG. 1 (color online). $t_{c} P(t)$ for normal diffusion with $\alpha=0$ (or $\beta_{\mathrm{CD}} \equiv 1-\alpha=1$ ). According to Eq. (3), the bending tail $t^{-1.5} \exp (-\Gamma t)$ at longer time is controlled by $\tau_{L, k}$ and the ratio between $E_{a, k}$ and the thermal energy.

mation from the above POP treatment. We have also obtained the same power law in Eq. (9) by COP treatment using a time-retarded diffusion kernel. Their slight differences occur at longer time when $1 / \Gamma_{k} \leq t \leq \tau_{L, k}$.

The experimental values for the exponent varies among research groups, some reported a value close to -1.5 , whereas others reported a larger value, e.g., Shimizu et al. (CdSe, $\left.m_{\text {on,off }}=-1.5, \mathrm{CdTe}, m_{\text {on,off }}=-1.6\right)$ [4] and (CdSe on gold substrate, $m_{\text {on,off }}=-1.4$ ) [5], Kuno et al. $\left(\mathrm{CdSe}, m_{\mathrm{off}}=-1.5\right.$ at $10 \mathrm{~ms} / \mathrm{bin},-1.6$ at $0.1 \mathrm{~ms} / \mathrm{bin})[1]$ and $\left(\mathrm{CdSe}, m_{\mathrm{on}}=-1.6, m_{\mathrm{off}}=-1.7\right)$ [7] and (InP, $m_{\text {on }=-2.0}, m_{\text {off }}=-1.5$ ) [3], Brokmann

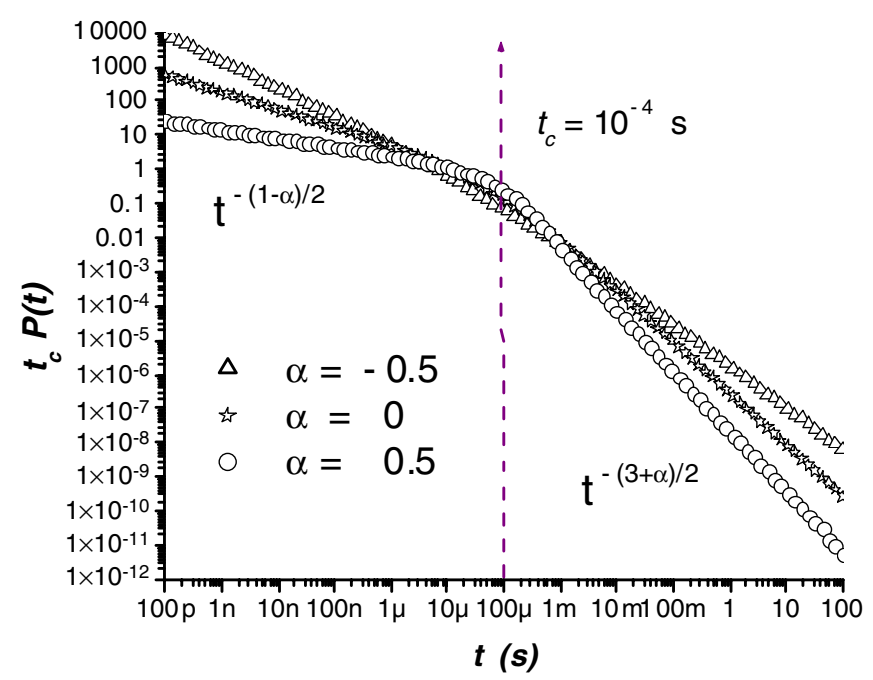

FIG. 2 (color online). $\quad t_{c} P(t)$ for several $\alpha$ 's, $\alpha$ defined as $1-$ $\beta_{\mathrm{CD}}$, showing two complementary power-law regimes, separated by the critical time constant $t_{c}$ (arbitrarily set at $0.1 \mathrm{~ms}$ as an example) with a sum of the corresponding exponents equal to 2 . et al. $\left(\mathrm{CdSe}, m_{\mathrm{on}}=-1.58, m_{\mathrm{off}}=-1.48\right)$ [8], Verberk et al. (CdSe, $m=-1.65)$ [6], Pelton et al. (CdSe, $m=$ -1.7) [9], Cichos et al. ( $\left.\mathrm{Si}, m_{\text {on }}=-2.2, m_{\text {off }}=-1.7\right)$ [10]. A more recent experimental study by Issac et al. [25] indicates a correlation of the exponent with dielectric properties as also suggested by this work. Anomalous diffusion is often observed in disordered systems [26].

In conclusion, the diffusion-controlled reaction mechanism is proposed as an underlying mechanism for fluorescence intermittency of QDs. It leads to a power law $P(t) \sim t^{m} \exp (-\Gamma t)$ and accommodates $m$ to be different from the ideal -1.5 due to the dispersive diffusion correlation times. The present model provides some physical links for the exponent $m=-2+\beta_{\mathrm{CD}} / 2$, the critical time $t_{c, k}$ (separating two complementary regimes) and the exponential bending tail $\exp \left(-\Gamma_{k} t\right)$, with $\Gamma_{k}$ related to energetic parameters (reorganization energy $\lambda$, free energy gap $\Delta G^{0}$, temperature) and kinetic parameters $\left(\tau_{L}\right.$ and $\left.\tau_{D}\right)$. A complementary power-law behavior with $m=-\beta_{\mathrm{CD}} / 2$ is predicted at a time shorter than $t_{c}$, where $t_{c}$ is controlled by the structural parameters such as electronic coupling $V_{k}$ and others. A power law has also been observed in single molecule Raman spectroscopy [27] and in protein conformation dynamics [28]. The possible extension of this diffusion-controlled reaction model to these areas remains to be explored.

The authors thank the support of the National Science Foundation and the Office of Naval Research. J. T. also acknowledges the support of the James W. Glanville Foundation at the California Institute of Technology.

\footnotetext{
*Corresponding author. Electronic address: jautang@caltech.edu ${ }^{\dagger}$ Corresponding author. Electronic address: ram@caltech.edu
}

[1] M. Kuno, D. P. Fromm, H. F. Hamann, A. Gallagher, and D. J. Nesbitt, J. Chem. Phys. 112, 3117 (2000).

[2] M. Kuno, D. P. Fromm, A. Gallagher, D. J. Nesbitt, O. I. Micic, and J. J. Nozik, Nano Lett. 1, 557 (2001).

[3] M. Kuno, D. P. Fromm, H. F. Hamann, A. Gallagher, and D. J. Nesbitt, J. Chem. Phys. 115, 1028 (2001).

[4] K. T. Shimizu, R. G. Neuhauser, C. A. Leatherdale, S. A. Empedocles, W. K. Woo, and M. G. Bawendi, Phys. Rev. B 63, 205316 (2001).

[5] K. T. Shimizu, W. K. Woo, B. R. Fisher, H. J. Eisler, and M. G. Bawendi, Phys. Rev. Lett. 89, 117401 (2002).

[6] R. Verberk, A. M. van Oijen, and M. Orrit, Phys. Rev. B 66, 233202 (2002).

[7] M. Kuno, D. P. Fromm, S. T. Johnson, A. Gallagher, and D. J. Nesbitt, Phys. Rev. B 67, 125304 (2003).

[8] X. Brokmann, J. P. Hermier, G. Messin, P. Desbiolles, J. P. Bouchaud, and M. Dahan, Phys. Rev. Lett. 90, 120601 (2003).

[9] M. Pelton, D. G. Grier, and P. Guyot-Sionnest, Appl. Phys. Lett. 85, 819 (2004). 
[10] F. Cichos, J. Martin, and C. von Borczyskowski, Phys. Rev. B 70, 115314 (2004).

[11] A. L. Efros and M. Rosen, Phys. Rev. Lett. 78, 1110 (1997).

[12] J. Wang and P. Wolynes, J. Chem. Phys. 110, 4812 (1999).

[13] R. Verberk and M. Orrit, J. Chem. Phys. 119, 2214 (2003).

[14] E. Barkai, Y. Jung, and R. Silbey, Annu. Rev. Phys. Chem. 55, 457 (2004).

[15] G. Margolin and E. Barkai, J. Chem. Phys. 121, 1566 (2004).

[16] J. Tang and R. A. Marcus, J. Chem. Phys. 123, 054704 (2005).

[17] L. D. Zusman, Chem. Phys. 49, 295 (1980).

[18] H. Sumi and R. A. Marcus, J. Chem. Phys. 84, 4894 (1986).

[19] T. Renger and R. A. Marcus, J. Chem. Phys. 116, 9997 (2002).

[20] J. T. Hynes, J. Phys. Chem. 90, 3701 (1986).
[21] C.J.F. Böttcher and P. Bordewijk, Theory of Electric Polarization (Elsevier, Amsterdam, 1978), Vol. II.

[22] R. Metzler, E. Barkai, and J. Klafter, Phys. Rev. Lett. 82, 3563 (1999).

[23] S. A. Empedocles and M. G. Bawendi, J. Phys. Chem. B 103, 1826 (1999).

[24] A. Erdélyi, W. Magnus, F. Oberhettinger, and F. G. Tricomi, Higher Transcendental Functions (Krieger, New York, 1981), Vol. 1.

[25] A. Issac, C. von Borczyskowski, and F. Cichos, Phys. Rev. B 71, 161302(R) (2005).

[26] J. P. Bouchaud and A. Georges, Phys. Rep. 195, 127 (1990).

[27] A. R. Bizzarri and S. Cannistraro, Phys. Rev. Lett. 94, 068303 (2005).

[28] H. Yang, G. Luo, P. Karnchanaphanurach, T. Louie, I. Rech, S. Cova, L. Xun, and X.S. Xie, Science 302, 262 (2003). 